Chemical Physics 135 (1989) 375-380

North-Holland, Amsterdam

\title{
ELECTRON DELOCALIZATION IN TRINUCLEAR IRON-SULFUR CLUSTERS FROM DESULFOVIBRIO GIGAS
}

\author{
S.A. BORSHCH and L.F. CHIBOTARU \\ Institute of Chemistry, Academy of Sciences of the Moldavian SSR, Grosul Street 3, Kishinev 277028, USSR
}

Received 27 February 1989

\begin{abstract}
The origin of the distribution of an "excess" electron in the trinuclear mixed-valence cluster from Desulfovibrio gigas is discussed. The model used takes into account Heisenberg exchange and resonance interaction between all centres leading to the double exchange. It is shown that electron delocalization over two centres in the ground state is possible in a high-symmetry trimer due to vibronic effects. The same electron distribution also exists in a slightly distorted trimer. In the last case approximately equal population of all centres in the first excited state is possible. These results agree with experimental data.
\end{abstract}

\section{Introduction}

The trinuclear iron-sulfur cluster, presumably of the $\mathrm{Fe}_{3} \mathrm{~S}_{4}$ type, is an active centre of the ferredoxin II (FdII) from sulphat-reducing bacteria Desulfovibrio gigas [1]. This cluster exists in two oxidation states. In the oxidized form three ferric ions are in high-spin states with spin $S_{0}=5 / 2$. The antiferromagnetic exchange interaction leads to the total spin of a cluster $S=1 / 2$.

In the reduced form the cluster has the $\mathrm{Fe}$ (III)-Fe(III)-Fe(II) composition, i.e. it is mixed-valence cluster. The main problem in the theory of mixed-valence clusters is the problem of "extra" electron delocalization. As it has been shown in EPR and Mössbauer experiments [1,2], in the ground state of reduced FdII the "extra" electron is delocalized over two iron ions. The spin-coupling model was proposed [2], which takes into account the Heisenberg exchange interaction between three centres and "extra" electron delocalization over two centres resulting in a double exchange interaction [3]. The double exchange couples the high-spin ferric ion with the high-spin ferrous ion with spin $S_{0}=2$ and leads to the ferromagnetic ground state of the pair with spin equal to $9 / 2$. The antiferromagnetic interaction of the pair with the third ferric ion gives the resulting total spin $S=2$, which was observed in experiments $[2,4]$.

The model considering the delocalization only over two centres is based on an implicit assumption that one ion in a cluster is distinguished by some way. Furthermore, the difference between ions must be sufficiently noticeable thus tending to negligible resonance interaction with the distinguished ion. Unfortunately $\mathrm{X}$-ray crystallographic data for FdII are not available. However, some results indicate only minor structural differences between iron ions. In particular, the formation of the $\mathrm{Fe}_{3} \mathrm{~S}_{4}$ cluster from a cubane $\mathrm{Fe}_{4} \mathrm{~S}_{4}$ cluster is assumed to occur by simple removing of one iron atom. In reality the symmetry of $\mathrm{Fe}_{4} \mathrm{~S}_{4}$ is not $T_{d}$ but $D_{2 d}$ [6] but in this case the difference in structure of nearest surroundings of iron atoms is rather small too. It will be also noted that three exchange parameters in the oxidized FdII differ in no more than 20\% [7]. So the distribution of valence states observed in experiment [1] must arise from the electronic delocalization over three centres.

Besides the structural inequivalence, another factor influencing the type of electronic distribution in a cluster is the vibronic interaction. As it was shown earlier [8], the electronic distribution in high-symmetry trimers has some peculiarities. If the resonance interaction parameter is negative three types of electronic distribution in the ground state are possible: (1) the localization of the "extra" electron on one of the centres; (2) equal delocalization over three metallic centres; (3) the coexistence of localized and delocalized electronic distributions. 
In the excited state of a cluster the delocalization over two centres is realized. If the sign of the resonance interaction is positive only the one-centre localization is possible. However, all these results are valid only for a case of one electron in the field of spinless cores. In our case the "extra" electron interacts with three ferric ions with spins $S_{0}=5 / 2$.

In the present paper the possible origin of electronic distributions observed in FdII will be considered. With this end in view the calculation of the electronic energy spectrum will be performed and, where it will be necessary, the vibronic interaction will be taken into account.

\section{High-symmetry trimer}

There are two approaches to the calculation of the electronic energy spectra of exchange-coupled mixed-valence clusters. One of them is based on the construction of multielectron wavefunctions with fixed electron localization having necessary permutation symmetry. Then the linear combinations of three functions transforming under representations of the cluster symmetry group are formed [9]. In the case of direct exchange, one can obtain in this way analytical expressions for resonance and exchange splittings. However, as in mixedvalence clusters electron transfer and exchange occur through a bridge, the use of the generalized AndersonHasagava model [10] is more convenient. The Hamiltonian of this model may be written in the following form:

$$
\hat{\mathscr{H}}_{\mathrm{AH}}=\left(\begin{array}{lll}
-2 J_{0} \hat{S}_{1} \hat{s}-2 J_{2} \hat{S}_{2} \hat{S}_{3} & B \hat{\mathbf{I}} & B \hat{\mathbf{I}} \\
-2 J_{1}\left(\hat{S}_{1}+\hat{s}\right)\left(\hat{S}_{2}+\hat{S}_{3}\right) & & \\
B \hat{\imath} & -2 J_{0} \hat{S}_{2} \hat{s}-2 J_{2} \hat{S}_{1} \hat{S}_{3} & B \hat{\mathbf{I}} \\
& -2 J_{1}\left(\hat{S}_{2}+\hat{s}\right)\left(\hat{S}_{1}+\hat{S}_{3}\right) & \\
B \hat{\mathbf{I}} & B \hat{\mathbf{I}} & -2 J_{0} \hat{S}_{3} \hat{s}-2 J_{2} \hat{S}_{1} \hat{S}_{2} \\
& & -2 J_{1}\left(\hat{S}_{3}+\hat{s}\right)\left(\hat{S}_{1}+\hat{S}_{2}\right)
\end{array}\right)
$$

Here $J_{0}$ is the intra-atomic exchange integral, $J_{2}$ is the exchange integral between ions without "extra" electron (that is $\mathrm{Fe}$ (III) ions), $J_{1}$ is the same between the heterovalent ions, $B$ is the parameter of the resonance intercentre interaction, î is a unit matrix, $\hat{S}_{1}, \hat{S}_{2}$, and $\hat{S}_{3}$ are the spin operators for the ionic cores $\left(S_{1}=S_{2}=S_{3}=S_{0}=5\right.$ / $2), \hat{s}$ is the spin of the migrating electron. The intra-atomic exchange is introduced in Hamiltonian (1) to take into account the difference in energies of one-centre states with spins equal to $S_{0}+1 / 2$ and $S_{0}-1 / 2$ respectively. The interaction of these states for the ions with less than half-filled shell can essentially change the energy spectrum [10]. However, as for the $\mathrm{Fe}$ (II) ion the electronic configuration is $\mathrm{d}^{6}$ and only state with spin $S_{0}-1 / 2=2$ exists, the term proportional to $J_{0}$ can be dropped.

If we work in the basis of states with the definite total spin, the matrix Hamiltonian may be written independently for each possible value of the total spin $S$ and its projection $m$. In the absence of the magnetic field, the matrices for different $m$ coincide. One can pass to the basis in which not only $S$ but also an intermediate spin $S_{i j}$ $\left(S_{i j}=S_{i}+S_{j}\right.$, where $S_{i}$ and $S_{j}$ are the spins of centres without "extra" electron) is a good quantum number. Under this transformation, the diagonal blocks of Hamiltonian (1) become diagonal matrices whose elements are the eigenvalues of Heisenberg Hamiltonian. The unit matrix in nondiagonal blocks is replaced by the matrix of the corresponding $9 j$-symbols. The dimension of each block is equal to the number of possible values of intermediate spin.

The vibronic interaction with non-totally symmetric vibrations is the only possible reason of the nonhomogeneous electronic distribution in a high-symmetry mixed-valence system. Doubly degenerate e-vibrations composed of totally symmetric displacements of near-neighbour surrounding of the metal ions $Q_{i}(i=1,2,3)$ are active for a trimer. The electronic energy levels dependent on these distortions are the eigenvalues of the following matrix 


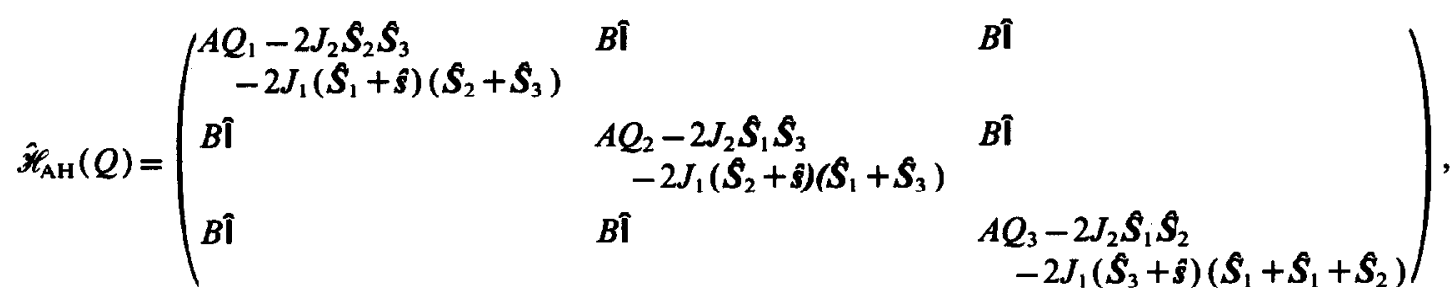

where $A$ is the constant of the electron-vibration interaction. The expressions for $Q_{i}$ in terms of symmetrized cluster vibrations are

$Q_{1}=(1 / \sqrt{3}) Q_{\mathrm{A}}+\sqrt{2 / 3} Q_{\theta}, \quad Q_{2}=(1 / \sqrt{3}) Q_{\mathrm{A}}-(1 / \sqrt{6}) Q_{\theta}+(1 / \sqrt{2}) Q_{t}$,

$Q_{3}=(1 / \sqrt{3}) Q_{\mathrm{A}}-(1 / \sqrt{6}) Q_{\theta}-(1 / \sqrt{2}) Q_{\epsilon}$.

Here $Q_{\mathrm{A}}$ is the normal vibration, transforming according to the totally symmetric a representation, $Q_{\theta}$ and $Q_{\epsilon}$ transform as the $\theta$ and $\epsilon$ components of the e representation. It can be easily seen that interaction with $A$ vibrations may be separated. It is known from experiment $[2,4]$ that the ground state has $S=2$ and no excited states with other spin lie below $80 \mathrm{~cm}^{-1}$. So only spin quintets originating from states with intermediate spins $S_{i j}=0$, $1,2,3,4$ will be considered below. The dimension of the resulting matrix will be $15 \times 15$. First functions of a basis correspond to the "extra" electron localization at first centre, etc.

If the difference of exchange integrals is neglected, i.e. $J_{1}=J_{2}=J$, one can obtain the following electronic energy levels at point $Q_{\theta}=Q_{\epsilon}=0$

$$
\begin{aligned}
& \epsilon\left({ }^{5} A^{(1)}\right)=\epsilon\left({ }^{5} E^{(1)}\right)=\epsilon\left({ }^{5} E^{(2)}\right)=-B, \quad \epsilon\left({ }^{5} A^{(2)}\right)=\epsilon\left({ }^{5} A^{(3)}\right)=\epsilon\left({ }^{5} E^{(3)}\right)=0, \\
& \epsilon\left({ }^{5} A^{(4)}\right)=\epsilon\left({ }^{5} A^{(5)}\right)=\epsilon\left({ }^{5} E^{(4)}\right)=\epsilon\left({ }^{5} E^{(5)}\right)=\frac{5}{6} B .
\end{aligned}
$$

All energies are read off a position of the unsplit Heisenberg multiplet with $S=2 \#$. Which of the energy levels belongs to which representation is defined through their transformation properties. So for a mixed-valence trimer we have five orbital singlets and five orbital doublets with $S=2$. For dimeric mixed-valence systems for arbitrary value of spin there are the same electronic levels, and therefore the same adiabatic potentials, as for the simplest $d^{0}-d^{1}$ dimer. The problem of calculation of adiabatic potentials for multielectron trimers and clusters of higher nuclearity is not reduced to those for one-electron systems.

In semiclassical approximation the electronic distribution is given by the adiabatic wavefunction in minima of an adiabatic potential. To obtain adiabatic potentials it is necessary to add the elastic energy $\frac{1}{2} \omega^{2}\left(Q_{\theta}^{2}+Q_{\epsilon}^{2}\right)$ ( $\omega$ is the frequency of e-vibrations) to the eigenvalues of matrix (2). For arbitrary $J_{1}, J_{2}, Q_{\theta}, Q_{t}$ the numerical diagonalization of $(2)$ is needed.

It is convenient to investigate the shape of adiabatic surface in polar coordinates

$Q_{\theta}=\rho \cos \varphi, \quad Q_{\epsilon}=\rho \sin \varphi$.

As can be seen from eqs. (4), the level scheme depends on the sign of the parameter $B$. However, qualitative behaviour of the lowest sheet of the adiabatic potential is the same for positive and negative $B$ and depends on the dimensionless parameter

$\Delta=|B| \omega^{2} / A^{2}$.

\#1 The energies in (4) differ from those given in ref. [9] due to the fact that non-realistic value of the Fe(II) spin $S_{0}+1 / 2=3$ is supposed in ref. [9]. 
As usually in the vibronic problem with active e vibrations, the adiabatic potential has threefold symmetry axis. For small $\Delta$ the minima of the lowest sheet of the adiabatic potential exist along directions $\frac{1}{3} \pi, \pi, \frac{5}{3} \pi$, and saddle points are along $\varphi=0, \frac{2}{3} \pi, \frac{4}{3} \pi$ directions. Each of the minima corresponds to the "extra" electron localization at one centre. For some two minima appear instead of one. They are situated symmetrically relative to the directions $\varphi=\frac{1}{3} \pi, \pi, \frac{5}{3} \pi$, which are now the saddle point directions. The value of $\Delta$ for which the number of minima increases twofold depends on the relationship between the exchange parameters. If $J_{1}-J_{2}=0-0.1$ (in $\hbar \omega$ units) appropriate $\Delta$ is in the interval $0.7-0.8$. The possibility of the six-minima surface in a vibronic problem with $\mathrm{e}$ vibrations was predicted by Dagys and Levinson [12]. Untill now such shape of adiabatic surface was obtained only for $\mathrm{d}^{0}$-hexafluoride complexes of transition metals [13]. The common feature of these two different problems is the vibronic mixing of more than two electronic terms.

Under further increasing of $\Delta$ the minima approach in pair to $\varphi=0, \frac{2}{3} \pi, \frac{4}{3} \pi$ directions, and merge. The electronic distribution in each of the new minima corresponds to the extra electron delocalization over two ferric ions. In the limit $\Delta \rightarrow \infty$, energies of minima and saddle points become equal and we have continuum of minima which is a trough. The motion along a trough corresponds to a dynamical delocalization of an electron which does not exist in dimeric mixed-valence systems.

Thus the appearance of electron delocalization over two centres in a high-symmetry trimer is possible due to vibronic effects.

\section{Slightly distorted trimer}

Consider a distribution of valence states in a slightly distorted trimer with electronic configuration $d^{5}-d^{5}-d^{6}$. The matrix Hamiltonian of the same type as (1) may be taken as a base for calculation of the electron energy spectrum. The distortion of a cluster leads to changes of energy of the orbital occupied by the extra electron on one centre, and to appearance of two parameters of resonance interaction. With these modification matrix (1) may be written as

$\hat{H}_{\mathrm{AH}}^{\mathrm{d}}=\left(\begin{array}{lll}\delta-2 J_{2} \hat{S}_{2} \hat{S}_{3} & B_{1} \hat{\imath} & B_{1} \hat{\mathbf{I}} \\ -2 J_{1}\left(\hat{S}_{1}+\hat{s}\right)\left(S_{2}+S_{3}\right) & & \\ B_{1} \hat{\imath} & -2 J_{2} \hat{S}_{1} \hat{S}_{3} & B \hat{\imath} \\ B_{1} \hat{\imath} & -2 J_{1}\left(\hat{S}_{2}+\hat{s}\right)\left(\hat{S}_{1}+\hat{S}_{3}\right) & \\ & B \hat{\mathbf{I}} & -2 J_{2} \hat{S}_{1} \hat{S}_{2} \\ & & -2 J_{1}\left(\hat{S}_{3}+\hat{s}\right)\left(\hat{S}_{1}+\hat{S}_{2}\right)\end{array}\right)$

where $\delta$ is the energy of destabilization on the first centre. The conditions $\delta>0$ and $B>B_{1}$ must be fulfilled in order to have delocalization over two centres in the ground state. The numerical calculation of the electron energy spectrum shows that delocalization over two centres appears in broad range of parameter values. However, the experimental Mössbauer spectrum [2] has some peculiarities which allow us to concretize the relationship between $B_{1}, B, \delta, \Delta J=J_{1}-J_{2}$. Above $20 \mathrm{~K}$ an additional quadrupole doublet other than the spectrum of the delocalized pair is observed. This new component corresponds to approximately uniform delocalization over all three centres. The origin of electronic state with about equal population of all three centres cannot be explained within the model with resonance interaction between two centres only [2]. We obtained that an excited state with nearly equal population of three local state lies close to the ground one provided that relations between the parameters are as follows

$B / B_{1} \approx 1.5, \quad \Delta J / B_{1} \approx \delta / B_{1} \approx 0.1$.

Thus for 
$B / B_{1}=1.5, \Delta J / B_{1}=\delta / B_{1}=0.125$

we have populations in the ground state

$C_{1}=0.02, \quad C_{2}=C_{3}=0.49$

and in the first excited state

$C_{1}=0.28, \quad C_{2}=C_{3}=0.36$.

Mössbauer spectroscopy does not allow one to split components with small difference in the populations of local electronic states. Thus the width of the doublet originating from the excited state must be slightly larger.

Next excited states also correspond to approximately uniform distribution of an excess electron over metallic sites. Thus the double exchange model with small distortions allows us to explain the appearance of additional doublet with delocalization over three centres as well as the origin of the low-temperature spectra with delocalization over two centres.

Consideration of vibronic interaction for a distorted trimer requires an approach differing from that given in section 2. All three normal vibrations becomes active in electron transfer [14]. However, as all electronic levels in this case are nondegenerate, for not very strong vibronic coupling the minima of the adiabatic potential are located in points with zero value of non-totally symmetric normal coordinates. The electronic distribution in these points is the same as in the absence of vibronic coupling.

\section{Conclusion}

As can be seen from the previous consideration, the Mössbauer data for the trinuclear iron-sulfur mixedvalence cluster from Desulfovibrio gigas can be explained without assumption about essential non-equivalence of one of the iron atoms. It is possible to have delocalization over two ions in a trimer with small distortions, or even in a highly symmetrical trimer due to the effect of vibronic interaction. For a distorted trimer the low-lying excited state with nearly equal population of all metallic centres, which was observed experimentally, is possible. The origin of this excited state in a highly symmetrical trimer (if it has the same spin as the ground one) remains unclear.

The results obtained are important for general theory of multinuclear mixed-valence clusters. As it was shown earlier purely electronic approach is invalid for the description of magnetic properties of mixed-valence trimers [15]. Now we can see that common consideration of exchange and vibronic interactions can lead to new effects in electronic distribution. These effects are impossible in a cluster with spinless cores.

\section{Acknowledgement}

The authors wish to thank Professor E. Münck and Dr. J.-J. Girerd who brought the results related to FdII to their attention.

\section{References}

[1] B.H. Huynh, J.J.G. Moura, I. Moura, T.A. Kent, J. Le Gall, A.V. Xavier and E. Münck, J. Biol. Chem. 255 (1980) 3242.

[2] V. Papaefthymiou, J.J. Girerd, I. Moura, J.J.G. Moura and E. Münck, J. Am. Chem. Soc. 109 (1987) 4703.

[3] P.W. Anderson and M. Hasegawa, Phys. Rev. 100 (1955) 675.

[4] A.J. Thomson, A.E. Robinson, M.K. Johnson, J.J.G. Moura, I. Moura, A.V. Xavier and J. Le Gall, Biochim. Biophys. Acta 670 (1981) 93. 
[5] J.J.G. Moura, I. Moura, T.A. Kent, J.D. Lipskomb, B.H. Huynh, J. Le Gall, A.V. Xavier and E. Münck, J. Biol. Chem. 257 (1982) 6259.

[6] L. Noodleman, J.G. Norman Jr., J.H. Osborne, A. Aizman and D.A. Case, J. Am. Chem. Soc. 107 (1985) 3418.

[7] J.-P. Gayda, P. Bertrand, F.-X. Theodule and J.J.G. Moura, J. chem. Phys. 77 (1982) 3387.

[8] S.A. Borhsch, I.N. Kotov and I.B. Bersuker, Chem. Phys. Letters 89 (1982) 381.

[9] M.I. Belinskii, Mol. Phys. 60 (1987) 793.

[10] S.A. Borhsch, Fiz. Tverd. Tela 29 (1987) 1561, in Russian.

[11] S.A. Borshch, I.N. Kotov and I.B. Bersuker, Chem. Phys. Letters 111 (1984) 264.

[12] R.S. Dagys and I.B. Levinson, Opt. i Spectroskopiya 3 (1967) 3, in Russian.

[13] S.A. Borshch, I.Ya. Ogurtsov and I.B. Bersuker, Zh. Struct. Khim. 23 (1983) 7, in Russian.

[14] S.A. Borshch and I.N. Kotov, Teor. i Eksper. Khimia 25 (1989) 129, in Russian.

[15] 1.B. Bersuker, S.A. Borshch and I.N. Kotov, Rev. Roum. Chim. 32 (1987) 1075. 